\title{
Dynamic Denisyuk holograms in cubic photorefractive crystals
}

\author{
S.M. Shandarov, N.I. Burimov, Yu.N. Kul'chin, R.V. Romashko, A.L. Tolstik, V.V. Shepelevich
}

\begin{abstract}
Theoretical and experimental studies of the interaction of two counterpropagating light waves on dynamic reflection gratings formed in the Denisyuk scheme in cubic photorefractive crystals due to the diffusion mechanism of the charge transfer are considered. The results are presented which demonstrate the possibility of using dynamic photorefractive Denisyuk holograms to design adaptive elements of measuring systems based on optical and fibreoptic interferometers.
\end{abstract}

Keywords: cubic photorefractive crystals, Denisyuk holograms, adaptive interferometers.

\section{Introduction}

Optical photorefractive nonlinearity is observed in crystals without the symmetry centre at laser radiation intensities $\sim 1 \mu \mathrm{W} \mathrm{mm}{ }^{-2}$. Two-wave and four-wave processes of light self-action caused by the photorefractive nonlinearity are accompanied by the formation of volume holograms in the crystal and can be used in optical memory, the amplification and phase conjugation of light beams and holographic interferometry [1-5]. The dynamic character of produced three-dimensional phase holograms allows one, for example, to use two-wave interaction for the adaptive correlation filtration of speckle patterns of the light field in precision optical sensors [6-8]. Cubic photorefractive crystals belonging to noncentrally symmetric classes 23 [sillenites $\mathrm{Bi}_{12} \mathrm{SiO}_{20}$ (BSO), $\mathrm{Bi}_{12} \mathrm{GeO}_{20}$ (BGO), $\mathrm{Bi}_{12} \mathrm{TiO}_{20}$ (BTO)] and $\overline{4} 3 m$ (high-resistance GaAs, GaP, InP, CdTe semiconductors) are attractive for such applications due to their fast response and resistance to external factors (temperature, humidity, vibrations, etc.). Many parameters of these

S.M. Shandarov, N.I. Burimov Tomsk State University of Control Systems and Radioelectronics, prosp. Lenina 40, 634050 Tomsk, Russia; e-mail: bni@fet.tusur.ru;

Yu.N. Kul'chin, R.V. Romashko Institute for Automation and Control Processes, Far-Eastern Branch, Russian Academy of Sciences, ul. Radio 5, 690041 Vladivostok, Russia; e-mail: kulchin@hq.febras.ru;

A.L. Tolstik Belarusian State University, prosp. Nezavisimosti 4, 220030 Minsk, Belarus; e-mail: tolstik@bsu.by;

V.V. Shepelevich I.P. Shamyakin Mozyr State Pedagogical University, ul. Studencheskaya 28, 247760 Mozyr, Belarus; e-mail: vasshep@inbox.ru

Received 14 January 2008; revision received 11 April 2008 Kvantovaya Elektronika 38 (11) 1059-1069 (2008)

Translated by I.A. Ulitkin crystals can be found in [1-5] as well as in recent review [9].

To increase the diffraction efficiency of the transmission hologram produced in the conventional scheme of interaction between two copropagating beams in a cubic crystal with small electrooptical coefficients $\left(r_{41} \sim 1-5 \mathrm{pm} \mathrm{V}^{-1}\right)$, the external electric field strength should exceed $10 \mathrm{kV} \mathrm{cm}^{-1}$ [1-9]. In the absence of the external field, a significant photorefractive response in cubic crystals can be achieved upon the interaction of counterpropagating light waves on a volume reflection hologram formed in the Denisyuk scheme $[10,11]$. These papers substantiate the principles and geometry of writing and reading reflection holograms, describe their main properties and point out their possible applications.

The interaction of light waves on reflection gratings in photorefractive crystals, in particular, BSO and BGO, was analysed theoretically and numerically (for example, in papers $[12-16])$ by neglecting, however, the natural optical activity inherent in these cubic crystals. The effect of the optical activity on the features of reflection holograms in cubic photorefractive (001) crystals was first studied in papers [17, 18]. The dependence of the output energy parameters of the hologram (the diffraction efficiency and relative intensity of the signal wave during the twowave interaction) on the crystal thickness was obtained and studied in [18]. It was shown that when the modulus of the coupling constant was smaller than the specific rotation of the crystal, the dependence of the output energy parameters of the hologram on the crystal thickness was oscillatory.

The first experimental studies $[16,19-22]$ demonstrated the possibility of producing photorefractive Denisyuk holograms in BGO, BTO and BSO crystals of the (100) and (111) cuts. The maximum photorefractive response was observed in doped $\mathrm{Fe}, \mathrm{Cu}$ : BTO samples (the diffraction efficiency of a hologram exceeded $60 \%$ ) [20] and Cd : BTO samples (the two-beam gain achieved $1.33 \mathrm{~cm}^{-1}$ ) [22] in the form of plates cut normal to the [100] crystallographic direction [the (100) cut]. The authors of papers [21, 22] revealed the vector character of the interaction of counterpropagating waves in the (100) cut, which consisted in the influence of the pumpbeam intensity and orientation of its polarisation vector on the amplitude and polarisation vector of the weak signal beam. The simple theoretical model of this interaction in the (100) crystal [22] taking into account the optical activity and light absorption was consistent with the experimental results.

The vector interaction of light waves on phase reflection gratings in photorefractive sillenite crystals of an arbitrary 
cut was considered in paper [23] in the undepleted pump approximation. Apart from the natural optical activity and absorption of light, the photoelastic contribution to the phase modulation of the crystal optical properties by the spatial charge field of the grating was taken into account in [23]. The method taking into account the photoelastic contribution for photorefractive gratings in cubic piezoelectric crystals was described, for example, in papers [24-26]. Explicit expressions for the components of the inverse permittivity tensor in cubic photorefractive crystals for an arbitrary orientation of the holographic grating vector were obtained in [27] (they are also presented in [5], p. 229 in notations used in [27]).

The scalar model of the interaction of two counterpropagating linearly polarised waves taking into account the change in the pump power but neglecting the transformation of their polarisation vectors was used in [28] to analyse the formation dynamics of the phase reflection hologram in a (100) BTO crystal. Paper [29] was devoted to the theoretical and experimental study of the two-beam interaction of circularly polarised waves on the reflection grating having the phase and amplitude components in a (111) Ca, $\mathrm{Ga}$ : BTO crystal. Thus, the reflection hologram produced in the photorefractive crystal due to the diffusion mechanism of the charge redistribution over donor and trap defect centres can have, along with the phase component, the amplitude component as well.

Due to the trap saturation effect $[1,4,5]$, to achieve the maximum photorefractive response during the interaction of counterpropagating waves on reflection holograms, a crystal should have a high concentration of trap centres. Sillenite BSO and BTO crystals, in which the high efficiency of interaction of counterpropagating waves was demonstrated experimentally $[20,22,28-31]$, correspond to this condition.

It was found in [22] that the amplification of a signal by reflection holograms in a cubic optically active photorefractive crystal cut perpendicular to the [111] crystallographic axis was smaller than that in the crystal cut perpendicular to the [001] axis. This statement is valid for small crystal thicknesses. This is also valid for any practically achievable thicknesses of a cubic crystal of the $\overline{4} 3 m$ symmetry with the same parameters, in which the optical activity is forbidden by the symmetry rules. However, this statement is not valid for cubic photorefractive optically active crystals. Studies performed in [32] showed that for certain crystal thicknesses, the (111) cut is preferable than the (001) cut both to obtain a high signal gain and to optimise the diffraction efficiency. Note that the polarisation dependences of the two-beam amplification efficiency of the phase-modulated signal wave on the reflection photorefractive grating in three identical (111) BSO, BTO and BGO crystals were studied in detail in paper [33].

Of great practical interest is the problem of determining the maximum achievable polarisation-optimised output parameters of the reflection holograms written in cubic optically active crystals in the Denisyuk scheme and the study of the dependence of these parameters on the crystal thickness and cut. This problem was partially considered in [32] and more completely solved in papers [34-36] by using the indicating surface method. The indicating surfaces of the polarisation-optimised diffraction efficiency of reflection holograms in a cubic photorefractive optically active crystal were constructed and analysed in [35]. Similar surfaces for the relative intensity of the object wave were considered in $[34,36]$.

The possibility of the efficient use of the interaction of counterpropagating waves on reflection holograms in BSO and BTO crystals of the (111) and (100) cuts in holographic interferometry devices, for adaptive correlation filtration of dynamic speckle patters and in precision subnanometer control systems for positioning reflecting surfaces was demonstrated in [19, 20, 37-41].

Below, we consider in detail the papers devoted to the theoretical analysis and experimental studies of the interaction of counterpropagating light waves on dynamic reflection Denisyuk gratings produced in cubic photorefractive crystals due to the diffusion charge transfer and their use to construct adaptive holographic correlators and interferometers.

\section{General equations describing the interaction of two counterpropagating plane light waves}

The light fields of plane signal (S) and pump (P) waves with the wave normals $\boldsymbol{n}_{\mathrm{S}}$ and $\boldsymbol{n}_{\mathrm{P}}$ respectively (Fig. 1), which interact in a cubic crystal of symmetry 23 , in view of the natural gyrotropy inherent in the crystal, can be represented in the from of a superposition of circularly polarised waves $[23,28,29,31,42]$ :

$$
\begin{aligned}
& \boldsymbol{E}_{\mathrm{P}}(x)=\left[C_{\mathrm{P} 1}(x) \boldsymbol{e}_{1} \exp \left(-\mathrm{i} k_{0} n_{1} x\right)\right. \\
& \left.+C_{\mathrm{P} 2}(x) \boldsymbol{e}_{2} \exp \left(-\mathrm{i} k_{0} n_{2} x\right)\right] \exp (-\alpha x / 2), \\
& \boldsymbol{E}_{\mathrm{S}}(x)=\left[C_{\mathrm{S} 1}(x) \boldsymbol{e}_{1}^{*} \exp \left(\mathrm{i} k_{0} n_{1} x\right)\right. \\
& \left.\quad+C_{\mathrm{S} 2} \boldsymbol{e}_{2}^{*} \exp \left(\mathrm{i} k_{0} n_{2} x\right)\right] \exp (\alpha x / 2)
\end{aligned}
$$

where $e_{1,2}=\left(y^{0} \pm \mathrm{i} z^{0}\right) / \sqrt{2}$ are the unit vectors corresponding to the left and right circular polarisations; $n_{1,2}=n_{0}$ $\pm \rho / k_{0}$ are the refractive indices of the natural waves; $\boldsymbol{y}^{0}$ and $z^{0}$ are the unit vectors of the laboratory coordinate system (see Fig. 1); $k_{0}=2 \pi / \lambda$ is the wave number in the vacuum; $n_{0}$ and $\alpha$ are the refractive index and absorption coefficient of the unperturbed crystal, respectively, and $\rho$ is its specific optical rotation.

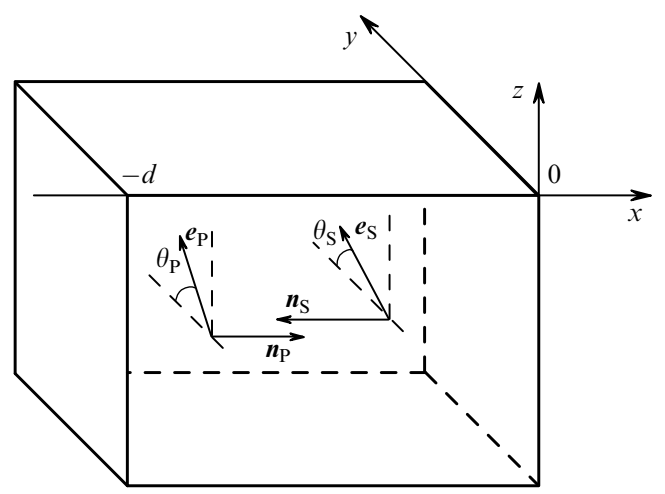

Figure 1. Geometry of the interaction of counterpropagating light signal (S) and pump (P) waves in a cubic gyrotropic photorefractive crystal. 
The interference pattern of the signal and pump waves producing a hologram in the crystal has the grating vector $\boldsymbol{K}=2 k_{0} n_{0} \boldsymbol{x}^{0}$, the contrast $m$ and the average intensity $I_{0}$ :

$$
\begin{gathered}
m(x)=2 \frac{C_{\mathrm{S} 1}(x) C_{\mathrm{P} 2}^{*}(x)+C_{\mathrm{S} 2}(x) C_{\mathrm{P} 1}^{*}(x)}{I_{0}(x)}, \\
I_{0}(x)=\left[\left|C_{\mathrm{P} 1}(x)\right|^{2}+\left|C_{\mathrm{P} 2}(x)\right|^{2}\right] \exp (-\alpha x) \\
+\left[\left|C_{\mathrm{S} 1}(x)\right|^{2}+\left|C_{\mathrm{S} 2}(x)\right|^{2}\right] \exp (\alpha x) .
\end{gathered}
$$

The photoexcitation of charge carriers exposed to nonuniform illumination is accompanied by their redistribution among defect centres. At the low contrast $(m \ll 1)$, the distribution of the volume charge produced in the absence of the external electric field due to the diffusion mechanism, contains only the first spatial harmonic with the vector $\boldsymbol{K}$ and the spatial period $\Lambda=\lambda / 2 n_{0}$ displaced by the quarter of this period with respect to the interference pattern [1-5]. The first harmonic amplitude of the electrostatic field produced by the volume charge is a linear function of the contrast

$$
E_{1}(x, t)=-\mathrm{i} m(x) E_{\mathrm{sc}}(t),
$$

and its formation dynamics is determined by the function $E_{\mathrm{sc}}(t)$ depending on the energy level diagram of defect centres. In the case of the single-level band model of a photorefractive crystal [43], the stationary value of this function

$$
\tilde{E}_{\mathrm{sc}}=\frac{E_{\mathrm{d}}}{1+E_{\mathrm{d}} / E_{q}}
$$

is determined by the diffusion field $E_{\mathrm{d}}=\left(k_{\mathrm{B}} T / e\right) K$ and the trap saturation field $E_{q}=e N_{\mathrm{a}} /(\varepsilon K)$, where $K=|\boldsymbol{K}| ; k_{\mathrm{B}}$ is the Boltzmann constant; $T$ is the temperature; $e$ is the elementary electric charge; $N_{\mathrm{a}}$ is the concentration of compensating acceptor centres; and $\varepsilon$ is the crystal static permittivity.

The modulation of the crystal refractive index by the spatial discharge field caused by the linear electrooptical effect and the combined action of the piezoelectric and photoelastic effects [5, 24-27], provides the phase (photorefractive) component of the dynamic hologram. Because of the complex structure of defect centres, photoinduced changes in the optical absorption [44], which contribute to the amplitude (absorption) component of this reflection hologram, can proceed in the crystal. In the linear approximation in contrast $m$, the amplitude of the first spatial harmonic of the absorption grating can be represented in the form $\Delta \alpha_{1}(x, t)=m(x) \alpha_{g}(t)$, where $\alpha_{g}(t)$ is the parameter characterising the spatially inhomogeneous photoinduced changes in the crystal absorption.

Taking into account the local relation of the absorption component of the hologram with the interference pattern and contributions of the electrooptical and photoelastic effects to the phase component, it is possible to obtain perturbations of the corresponding tensor components with respect to the permittivity in the from [31]

$$
\begin{gathered}
\Delta \varepsilon_{m n}(x, t)=\left[\Delta \varepsilon_{m n}^{\mathrm{ph}} \exp (\mathrm{i} K x)+\Delta \varepsilon_{m n}^{\mathrm{ph}^{*}} \exp (-\mathrm{i} K x)\right] / 2 \\
+\left[\Delta \varepsilon_{m n}^{\mathrm{abs}}(x, t, m) \exp (\mathrm{i} K x)+\Delta \varepsilon_{m n}^{\mathrm{abs}}\left(x, t, m^{*}\right) \exp (-\mathrm{i} K x)\right] / 2
\end{gathered}
$$

where

$$
\begin{gathered}
\Delta \varepsilon_{m n}^{\mathrm{ph}}=\mathrm{i} m\left(n_{0}^{4} E_{\mathrm{sc}} \Delta b_{m n}\right) ; \Delta \varepsilon_{m n}^{\mathrm{abs}}(m)=-\mathrm{i} m\left(\alpha_{g} \delta_{m n} n_{0} / k_{0}\right) ; \\
\Delta b_{m n}=\left[\left|\delta_{m n p}\right| p_{p}+\frac{P_{m n k l}^{E} p_{l} \gamma_{k i} e_{p i r} p_{p} p_{r}}{r_{41}^{S}}\right]
\end{gathered}
$$

$\delta_{m n}$ is the unit symmetric tensor of second rank; $\delta_{m n p}$ is the unit antisymmetric tensor of third rank; $p_{p}$ are the direction cosines of the grating vector $\boldsymbol{K} \| \boldsymbol{x}^{0} ; r_{41}^{S}$ and $P_{m n k l}^{E}$ are the components of the electrooptical tensor of a clamped crystal and the photoelastic tensor measured at a constant electric field; $\gamma_{k i}$ are the components of the tensor inverse to $\Gamma_{i k}=C_{i j k l}^{E} p_{j} p_{l} ; C_{i j k l}^{E}$ and $e_{p i r}$ are the tensor components of the elasticity moduli and piezoelectric constants.

The transformation of the energy and polarisation characteristics of the signal and pump waves interacting on the holographic grating under study is described by the coupled-wave equations [31]:

$$
\begin{gathered}
\frac{\mathrm{d} C_{\mathrm{S} 1}}{\mathrm{~d} x}=-\frac{\gamma}{4} m\left[g_{I}^{*} C_{\mathrm{P} 1} \exp (-\mathrm{i} 2 \rho x)\right. \\
\left.+\left(g_{E}-g_{\mathrm{abs}}\right) C_{\mathrm{P} 2}\right] \exp (-\alpha x), \\
\frac{\mathrm{d} C_{\mathrm{S} 2}}{\mathrm{~d} x}=-\frac{\gamma}{4} m\left[\left(g_{E}-g_{\mathrm{abs}}\right) C_{\mathrm{P} 1}\right. \\
\left.+g_{I} C_{\mathrm{P} 2} \exp (\mathrm{i} 2 \rho x)\right] \exp (-\alpha x), \\
\frac{\mathrm{d} C_{\mathrm{P} 1}}{\mathrm{~d} x}=-\frac{\gamma}{4} m^{*}\left[g_{I} C_{\mathrm{S} 1} \exp (\mathrm{i} 2 \rho x)\right. \\
\left.+\left(g_{E}+g_{\mathrm{abs}}\right) C_{\mathrm{S} 2}\right] \exp (\alpha x), \\
\frac{\mathrm{d} C_{\mathrm{P} 2}}{\mathrm{~d} x}=-\frac{\gamma}{4} m^{*}\left[\left(g_{E}+g_{\mathrm{abs}}\right) C_{\mathrm{S} 1}\right. \\
\left.+g_{I}^{*} C_{\mathrm{S} 2} \exp (-\mathrm{i} 2 \rho x)\right] \exp (\alpha x),
\end{gathered}
$$

where $\gamma=k_{0} n_{0}^{3} r_{41}^{S} E_{\mathrm{sc}}$ is the coupling constant.

The coefficients $g_{E}=\left(\boldsymbol{e}_{1}^{*} \Delta \boldsymbol{b} \boldsymbol{e}_{1}\right)=\left(\boldsymbol{e}_{2}^{*} \Delta \boldsymbol{b} \boldsymbol{e}_{2}\right)$ and $g_{\mathrm{abs}}=$ $\alpha_{\mathrm{g}} /\left(k_{0} n_{0}^{3} r_{41}^{S} E_{\mathrm{sc}}\right)$ in Eqns (10)-(13) characterise the contributions of the phase and absorption components of the hologram, respectively, to the intermode processes (with a change in the intrinsic refractive index) during the interaction of counterpropagating waves. The intramode interaction (without a change in the intrinsic refractive index), characterised by the coefficient $g_{I}=\left(\boldsymbol{e}_{1}^{*} \Delta \boldsymbol{b} \boldsymbol{e}_{2}\right)$, is determined only by the phase component of the given hologram. The contribution of the absorption component of the grating is isotropic and the coefficient $g_{\text {abs }}$ is independent of the direction of its vector $\boldsymbol{K}$, which is parallel to the unit vector $\boldsymbol{x}^{0}$ in the case under study: $\boldsymbol{K}=2 k_{0} n_{0} \boldsymbol{x}^{0}$. The anisotropy of the coefficients $g_{I}$ and $g_{E}$ determining the efficiency and polarisation characteristics of the interaction of the signal and pump waves on the reflection hologram was studied in detail for the BTO 
Table 1. Coefficients characterising the intermode $\left(g_{E}\right)$ and intramode $\left(g_{I}\right)$ processes for different directions of the pump-wave propagation in BTO and BSO crystals.

\begin{tabular}{|c|c|c|c|c|c|c|c|c|c|}
\hline \multicolumn{2}{|c|}{$\begin{array}{l}\text { Pump-wave } \\
\text { propagation } \\
\text { dirfection }[\mathrm{mnp}]\end{array}$} & [100] & {$[\overline{1} 00]$} & {$[1 \overline{1} 0]$} & {$[\overline{1} 10]$} & {$[111]$} & {$[\overline{1} \overline{1} \overline{1}]$} & {$[11 \overline{2}]$} & {$[\overline{1} \overline{1} 2]$} \\
\hline & $g_{E}$ & 0 & 0 & 0 & 0 & -0.266 & 0.266 & 0.216 & -0.216 \\
\hline \multirow[t]{2}{*}{ BSO } & $g_{I}$ & $-\mathrm{i}$ & $\mathrm{i}$ & 0 & 0 & 0 & 0 & 0.407 & -0.407 \\
\hline & $g_{E}$ & 0 & 0 & 0 & 0 & 0.235 & -0.235 & -0.130 & 0.130 \\
\hline
\end{tabular}

crystal in paper [31]. The coefficients $g_{I}$ and $g_{E}$ calculated for BTO and BSO crystals at different orientations by using data from $[23,31]$ are presented in Table 1.

The coupling constant entering Eqns (10)-(13), which determines the interaction efficiency on the reflection grating with a small spatial period, is restricted by the concentration $N_{\mathrm{a}}$ of traps among which the volume charge is redistributed in a photorefractive crystal. Under stationary conditions, we have $\tilde{\gamma}=k_{0} n_{0}^{3} r_{41}^{S} \tilde{E}_{\mathrm{sc}}$, where $\tilde{\gamma}$ is the effective coupling constant and the effective field of the spatial charge $\tilde{E}_{\text {sc }}$ is determined by relation (6). The dependence of the coupling constant $\tilde{\gamma}$ on the concentration $N_{\mathrm{a}}$ of acceptors for a photorefractive Denisyuk hologram produced at a wavelength of $633 \mathrm{~nm}$ in a BTO crystal $(\Lambda=123 \mathrm{~nm})$ is shown in Fig. 2.

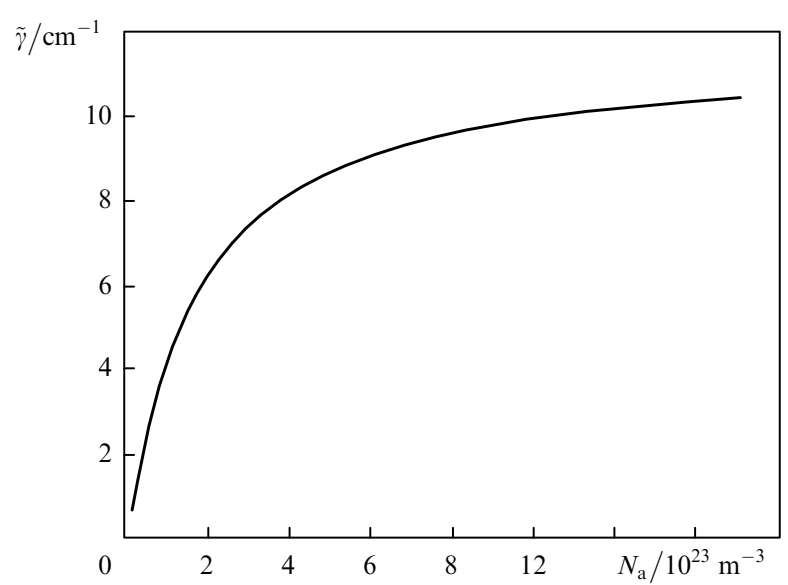

Figure 2. Dependence of the effective coupling constant $\tilde{\gamma}$ on the concentration of trap centres $N_{\mathrm{a}}$.

One can see that for BTO crystals with the acceptor concentration $N_{\mathrm{a}}=4 \times 10^{23} \mathrm{~m}^{-3}$, the coupling constant has the value $8 \mathrm{~cm}^{-1}$, which is achieved when the hologram is produced in 'transmission' only in the external electric field.

\section{Two-beam interaction of linearly polarised waves}

The amplitudes of linearly polarised interacting waves satisfy the conditions $C_{\mathrm{P} 1}(x)=C_{\mathrm{P} 2}^{*}(x)=C_{\mathrm{P}}(x)$ and $C_{\mathrm{S} 1}(x)=C_{\mathrm{S} 2}^{*}(x)=C_{\mathrm{S}}(x)$ and can be represented in the from:

$$
\begin{aligned}
& C_{\mathrm{P} 1,2}(x)=\left|C_{\mathrm{P}}(x)\right| \exp \left[\mp \mathrm{i} \varphi_{\mathrm{P}}(x)\right], \\
& C_{\mathrm{S} 1,2}(x)=\left|C_{\mathrm{S}}(x)\right| \exp \left[ \pm \mathrm{i} \varphi_{\mathrm{S}}(x)\right] .
\end{aligned}
$$

In this case, the passage to the intensities of the signal and pump waves in the crystal, $\tilde{I}_{\mathrm{S}}(x) \sim\left[\left|C_{\mathrm{S} 1}(x)\right|^{2}+\right.$ $\left.\left|C_{\mathrm{S} 2}(x)\right|^{2}\right] \exp (\alpha x) \quad$ and $\quad \tilde{I}_{\mathrm{P}}(x) \sim\left[\left|C_{\mathrm{P} 1}(x)\right|^{2}+\left|C_{\mathrm{P} 2}(x)\right|^{2}\right] \times$ $\exp (-\alpha x)$ allows one to obtain from $(10)-(13)$ the equations describing the interaction of linearly polarised counterpropagating waves in an arbitrarily oriented crystal [31]:

$$
\begin{aligned}
\frac{\partial \tilde{I}_{\mathrm{S}}}{\partial x} & =\alpha \tilde{I}_{\mathrm{S}}-\gamma\left[\left|g_{I}\right| \cos \left(2 \rho x+\varphi_{I}+\varphi_{\mathrm{S}}+\varphi_{\mathrm{P}}\right)\right. \\
& \left.+\left(g_{E}-g_{\mathrm{abs}}\right) \cos (\Delta \varphi)\right] \cos (\Delta \varphi) \frac{\tilde{I}_{\mathrm{S}} \tilde{I}_{\mathrm{P}}}{\tilde{I}_{\mathrm{S}}+\tilde{I}_{\mathrm{P}}}, \\
\frac{\partial \tilde{I}_{\mathrm{P}}}{\partial x} & =-\alpha \tilde{I}_{\mathrm{P}}-\gamma\left[\left|g_{I}\right| \cos \left(2 \rho x+\varphi_{\mathrm{I}}+\varphi_{\mathrm{S}}+\varphi_{\mathrm{P}}\right)\right. \\
& \left.+\left(g_{E}+g_{\mathrm{abs}}\right) \cos (\Delta \varphi)\right] \cos (\Delta \varphi) \frac{\tilde{I}_{\mathrm{S}} \tilde{I}_{\mathrm{P}}}{\tilde{I}_{\mathrm{S}}+\tilde{I}_{\mathrm{P}}}, \\
\frac{\partial \varphi_{\mathrm{S}}}{\partial x} & =\frac{\gamma}{2}\left[\left|g_{I}\right| \sin \left(2 \rho x+\varphi_{\mathrm{I}}+\varphi_{\mathrm{S}}+\varphi_{\mathrm{P}}\right)\right. \\
& \left.+\left(g_{E}-g_{\mathrm{abs}}\right) \sin (\Delta \varphi)\right] \cos (\Delta \varphi) \frac{\tilde{I}_{\mathrm{P}}}{\tilde{I}_{\mathrm{S}}+\tilde{I}_{\mathrm{P}}}, \\
\frac{\partial \varphi_{\mathrm{P}}}{\partial x} & =\frac{\gamma}{2}\left[\left|g_{I}\right| \sin \left(2 \rho x+\varphi_{\mathrm{I}}+\varphi_{\mathrm{S}}+\varphi_{\mathrm{P}}\right)\right. \\
& \left.-\left(g_{E}+g_{\mathrm{abs}}\right) \sin (\Delta \varphi)\right] \cos (\Delta \varphi) \frac{\tilde{I}_{\mathrm{S}}}{\tilde{I}_{\mathrm{S}}+\tilde{I}_{\mathrm{P}}},
\end{aligned}
$$

where $\Delta \varphi=\varphi_{\mathrm{S}}-\varphi_{\mathrm{P}} ; \varphi_{I}$ is the argument of the complex coefficient $g_{I}$. In the case of the negligibly small contribution of the absorption grating to the interaction, we obtain

$$
\begin{aligned}
& \tilde{I}_{\mathrm{S}}(x) \tilde{I}_{\mathrm{P}}(x)=\tilde{I}_{\mathrm{S}}(0) \tilde{I}_{\mathrm{P}}(0) \\
& \quad \times \exp \left\{-\gamma \int_{0}^{x}\left[\left|g_{I}\right| \cos \left(2 \rho x+\varphi_{I}+\varphi_{\mathrm{S}}+\varphi_{\mathrm{P}}\right)\right.\right. \\
& \left.\left.\quad+g_{E} \cos (\Delta \varphi)\right] \cos (\Delta \varphi) \mathrm{d} x\right\}
\end{aligned}
$$

from Eqns (15) and (16), which allows us to introduce the effective gain

$$
\Gamma_{\mathrm{eff}}=\frac{\gamma}{d} \int_{-d}^{0}\left[\left|g_{I}\right| \cos \left(2 \rho x+\varphi_{I}+\varphi_{\mathrm{S}}+\varphi_{\mathrm{P}}\right)\right.
$$

$$
\left.+g_{E} \cos (\Delta \varphi)\right] \cos (\Delta \varphi) \mathrm{d} x
$$


for the interaction of counterpropagating waves in a crystal of thickness $d$ [31].

This coefficient can be expressed through the intensities of the interacting waves, which can be determined from the experimental data:

$$
\Gamma_{\text {eff }}=\frac{1}{d} \ln \left\{\frac{\tilde{I}_{\mathrm{S}}(-d) \tilde{I}_{\mathrm{P}}(-d)}{\tilde{I}_{\mathrm{S}}(0) \tilde{I}_{\mathrm{P}}(0)}\right\} .
$$

The coefficient $\Gamma_{\text {eff }}$ characterises the efficiency of the vector interaction of counterpropagating waves on the phase reflection grating and is independent of light absorption in the crystal and of absorption changes proceeding during grating formation.

Analysis of the coefficients $g_{I}$ and $g_{E}$ presented in Table 1 shows that the efficiency of the two-beam interaction on the reflection Denisyuk hologram is maximum in the (100) crystals. However, the interaction maximum is observed when the polarisation vectors of light waves are oriented along the $\{011\}$ directions [23, 31]. Optical rotation due to the natural optical activity reduces the effective gain with increasing the crystal thickness. This is well observed in Fig. 3a borrowed from paper [31], where the results of the numerical integration of the system of equations (10)-(13) are presented for a (100)-cut bismuth titanate crystal with

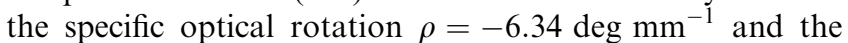
effective coupling constant $\tilde{\gamma}=6.8 \mathrm{~cm}^{-1}$ at $633 \mathrm{~nm}$. It was assumed in calculations that the reflection hologram was formed upon the interaction of the incident pump beam with the signal beam reflected from the sample output face $(x=0)$, while the input face $(x=-d)$ had an antireflection coating. In this case, the intensities and phases of the interacting waves for $x=0$ are related by the expressions $\tilde{I}_{\mathrm{S}}(0)=\tilde{I}_{\mathrm{P}}(0) R^{2}$ and $\varphi_{\mathrm{P}}(0)=\varphi_{\mathrm{S}}(0)=\theta_{\mathrm{P} 0}$, where $R$ is the Fresnel reflection coefficient for the normal incidence and $\theta_{\mathrm{P} 0}$ is the angle between the polarisation vector of the light field at the crystal output face and the $y$ axis of the coordinate system (Fig. 1). The dependences of the effective gain on the output angle $\theta_{\mathrm{P} 0}$ calculated by (21) for crystals with the thicknesses $d=0.1,2.6$ and $5.0 \mathrm{~mm}$ show that the gain maximum $\Gamma_{\text {eff }}=6.8 \mathrm{~cm}^{-1}$ is achieved at the minimum thickness $d=0.1 \mathrm{~mm}$ for $\theta_{\mathrm{P} 0}=135^{\circ}$. For $\theta_{\mathrm{P} 0}=45^{\circ}$, the gain achieves the same extreme but negative value corresponding to the most efficient power transfer from the signal beam to the pump beam. As the crystal thickness is increased, the amplitudes of extrema decrease and at negative values of the optical rotation they shift to the smaller values of the angle $\theta_{\mathrm{P} 0}$ the stronger the thicker the crystal.

Figure 3a shows that experimental data [31] obtained for a 2.6-mm-thick $\mathrm{Fe}, \mathrm{Cu}: \mathrm{Bi}_{12} \mathrm{TiO}_{20}$ crystal are consistent with the model of the interaction of counterpropagating plane light waves on a dynamic photorefractive hologram, and large gains can be achieved in bismuth titanate crystals without applying an external electric field. Experiments performed in $[28,30,31,42,45,46]$ showed that the effective gain depends on the stoichiometric composition and impurities contained in BTO crystals, and for the (100) crystals of thickness up to $10 \mathrm{~mm}$ the gain acquires the values from 1 to $7.4 \mathrm{~cm}^{-1}$.

Figure $3 b$ illustrates the change in the polarisation vectors of the signal and pump waves interacting on a dynamic hologram in the $\mathrm{Fe}, \mathrm{Cu}: \mathrm{Bi}_{12} \mathrm{TiO}_{20}$ crystal [31]. A
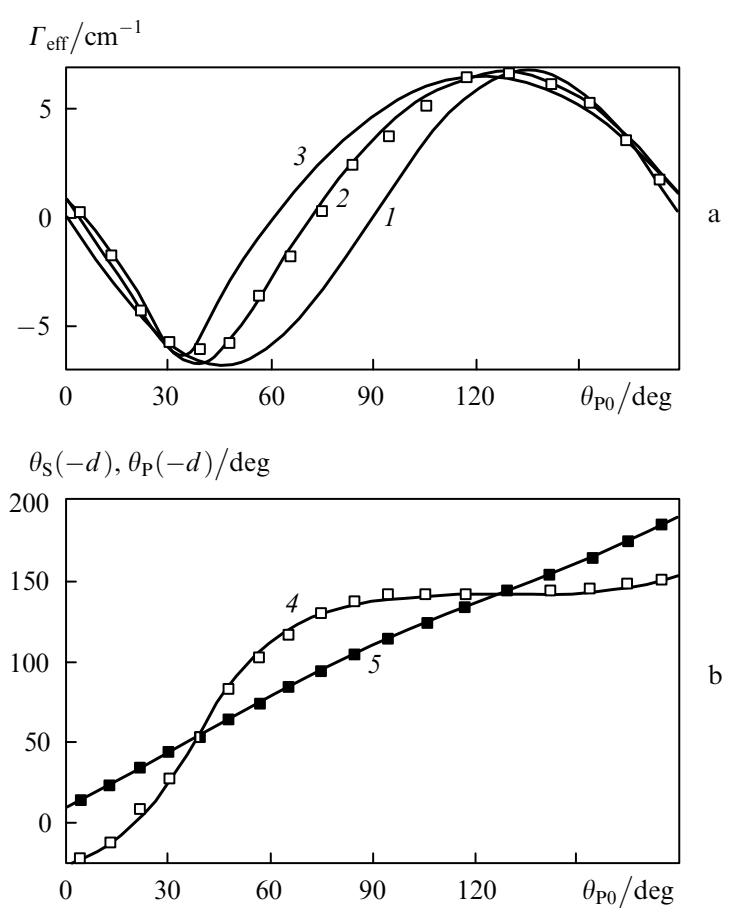

Figure 3. Dependences of the effective gain $\Gamma_{\text {eff }}$ for the interaction of counterpropagating waves (a) and polarisation angles $\theta_{\mathrm{S}}$ and $\theta_{\mathrm{P}}(\mathrm{b})$ of the signal (4) and pump (3) waves at the input face $x=-d$ on the angle $\theta_{\mathrm{P} 0}$, characterising the vector orientation of the light field polarisation at the output face $x=0$ for the $\mathrm{Bi}_{12} \mathrm{TiO}_{20}: \mathrm{Fe}, \mathrm{Cu}$ of thickness $d=0.1$ ( 1 ), $2.6(2,4,5)$ and $5.0 \mathrm{~mm}(3)$. Solid curves are the theory and points are the experiment.

comparison of the dependences of the polarisation angles $\theta_{\mathrm{P}}(-d)$ and $\theta_{\mathrm{S}}(-d)$, by which the pump and signal waves are characterised on the input face $x=-d$, on the 'output' angle $\theta_{\mathrm{P} 0}$ with the dependences $\Gamma_{\text {eff }}\left(\theta_{\mathrm{P} 0}\right)$ presented in Fig. 3a shows that the polarisations of these waves are similar for the extreme values of the gain. However, the maximum additional optical rotation of the signal wave due to the interaction on this reflection hologram is observed for $\Gamma_{\text {eff }}=0$.

As the gain decreases with increasing the crystal thickness, the $\{100\}$ cuts cease to be optimal at a certain crystal thickness $d>d_{k}[35,36]$. In this case, 'the optimal cut' is defined by the authors as a complex of symmetrically equivalent planes parallel to the working faces of the crystal with the fixed thickness and written phase reflection grating for which the polarisation-optimised diffraction efficiency $\eta_{\text {opt }}$ of the hologram achieves the maximum value $\eta_{\max }$.

Optimal crystal cuts of thickness $d>d_{k}$ are characterised by a family of planes with normals directed close to the $\{111\}$ directions in accordance with the triad axis $[35,36]$. As the crystal thickness is changed, the normals to the optimal-cut planes deviate slightly (the angle between the direction of any of them and the triad axis does not exceed $7.6^{\circ}$ for a BSO crystal), only in some cases [for $d=p \pi /(2 \rho)$, where $p=2,4, \ldots]$ degenerating into the triad axis of the type $\langle 111\rangle$.

The qualitative estimates of the dependences of the diffraction efficiency of the reflection hologram and the gain upon the two-wave interaction on the reflection hologram written in the BSO crystal on the thickness of the (001)- and (111)-cut crystals can be obtained by analysing the results obtained in [32]. 


\section{Adaptive holographic correlator on dynamic Denisyuk holograms}

Measuring systems based on optical or fibreoptic interferometers have a number of advantages, the most important among them being their high sensitivity. However, the high sensitivity under real conditions can be often undesirable, making the measuring system prone to the influence of the environmental parameters (temperature, mechanic stresses and vibrations, pressure, etc.). At the same time it is known that the two-wave mixing in photorefractive crystals can be used not only to transform efficiently the phase modulation of the optical interferometer radiation to the modulation of the output optical power but also to compensate for the low-frequency modulation caused by the change in the environmental conditions [6-8, 47-49].

By using the reflection scheme, it is possible, as mentioned above, to produce efficient dynamic holograms in photorefractive crystals containing a sufficient amount of photoactive trap centres without applying the external electric field to the crystal, and, hence, to simplify considerably measuring systems based on photorefractive holograms. The phase demodulation system based on such dynamic holograms can be constructed by using the speckle correlator or interferometer schemes.

The adaptive holographic correlator scheme in the photorefractive crystal can be constructed by using two light beams, one of which is obtained due to the partial reflection of a light wave reflected inside the crystal from its output face (Fig. 4a). If the initial light wave is obtained, for example, at the output of a multimode fibre (MMF) or due to scattering of coherent radiation from a rough surface and has a complicated speckle intensity distribution (see inset in Fig. 4a), the diffraction efficiency of a hologram produced by this wave will be a function of spatial coordinates in the plane perpendicular to the wave propagation direction. When such a hologram is read out by the initial speckle beam, diffracted waves appear whose power will be determined by the function of the spatial correlation between the intensity distributions of the speckle field and its diffraction efficiency. Thus, the change in the intensity distribution of the input light wave caused by the external interaction (being measured) will lead to the power modulation of the light wave reflected from the hologram.

In the case of a light wave emerged from the MMF, the change in the radiation power reflected from the hologram, caused by a change in the speckle pattern produced by the external action, is described by the expression [50]

$$
\Delta P_{\mathrm{R}}(t)=\frac{P_{\mathrm{R} 0}}{2 T} \int_{0}^{T} F(t, \tau) \exp \left(-\frac{\tau}{\tau_{\mathrm{R}}}\right) \mathrm{d} \tau,
$$

where

$$
F(t, \tau)=\frac{\sin ^{2}\{[\Delta \Phi(t)-\Delta \Phi(t-\tau)] / 2\}}{\{[\Delta \Phi(t)-\Delta \Phi(t-\tau)] / 2\}^{2}} ;
$$
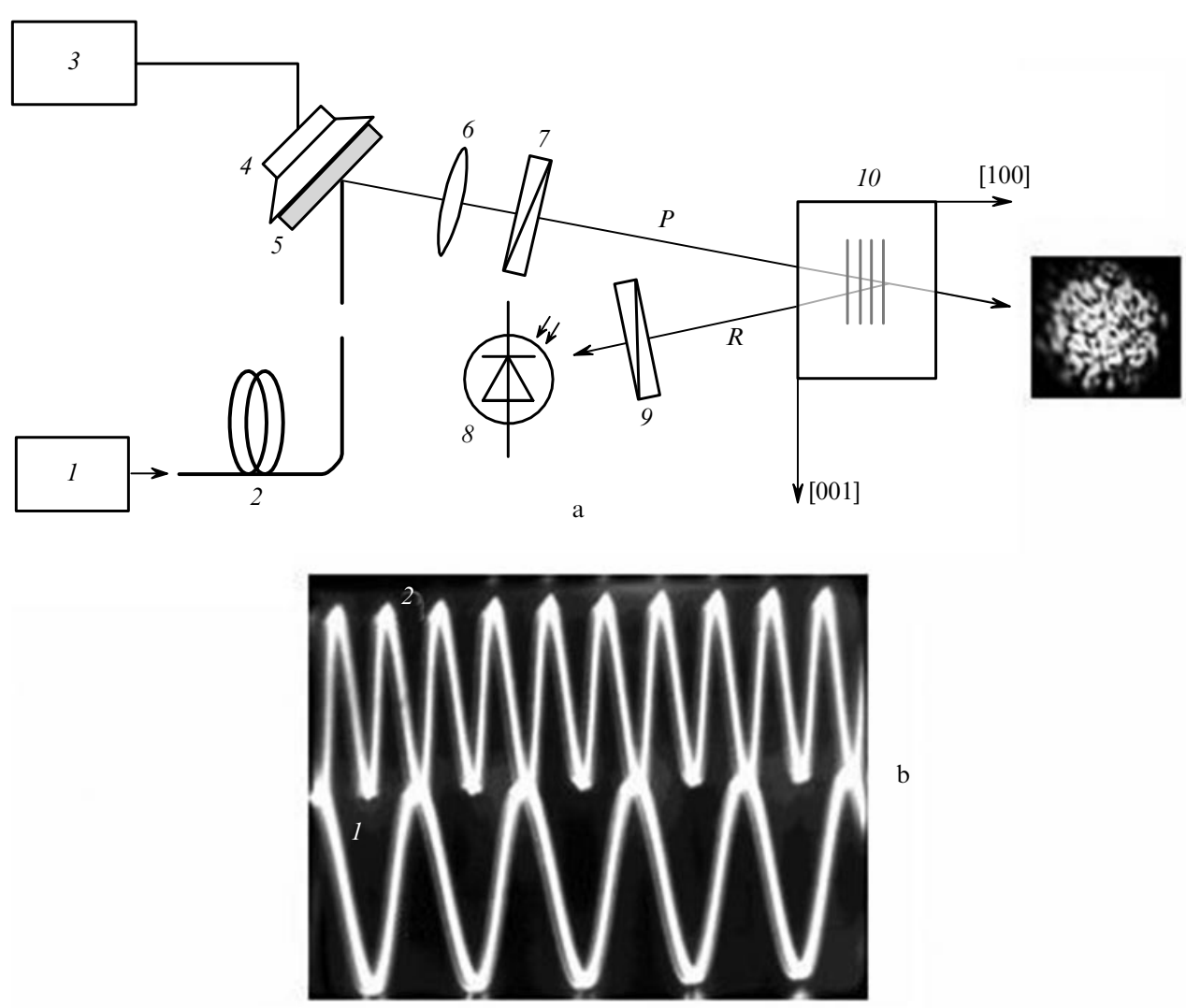

Figure 4. Scheme of the adaptive speckle-correlation measuring system based on the dynamic Denisyuk hologram (a) and oscillograms of the modulation signal ( 1 ) and the photodetector signal measuring the radiation power reflected from the hologram (2) (b): (1) laser; (2) multimode fibre (MMF); ( 3 ) sound generator (SG); (4) loudspeaker; ( 5 ) mirror; (6) lens; ( 7 ) polariser; (8) photodetector (PD); (9) analyser; (10) photorefractive $\mathrm{Bi}_{12} \mathrm{TiO}_{20}$ (BTO) crystal; $P$ is the input light wave (pump wave); $R$ is the reflected light wave (signal wave); the top photograph shows the intensity distribution of the radiation field at the MMF output. 
$\tau_{\mathrm{R}}$ is the writing time of the hologram; $P_{\mathrm{R} 0}$ is the radiation power diffracted from the reflection hologram, corresponding to the established regime $\left(t \gg \tau_{\mathrm{R}}\right) ; \Delta \Phi$ is the phase difference between the MMF modes, changing due to the external action; and $T$ is the characteristic time (period) of the phase difference variation.

Note that, if the longitudinal size of speckles is smaller than the photorefractive crystal thickness, the chaotic changes in the speckle pattern typical of MMFs will not cause the power modulation of the diffracted radiation due to the statistic averaging of successive contributions to the power variation in different 'layers' of the volume hologram. In the case of determined variations in the speckle pattern, for example, during the reflection of a wave containing the speckle pattern from an oscillating mirror turned to it by the angle $45^{\circ}$ (Fig. 4a), the demodulation signal will be detected. Figure $4 \mathrm{~b}$ presents the shape of the demodulation signal after the introduction of the harmonic phase modulation $\Delta \Phi=\Phi_{0} \sin \Omega t$ to the initial wave. The change in the diffracted radiation power is observed in this case at the second-harmonic frequency of the modulation signal, in agreement with expression (22) in which the integrand is an even function of the phase difference $\Delta \Phi$.

As mentioned above, the gain in the interaction of two counterpropagating waves in (100)-cut crystals, which determines the sensitivity of the adaptive correlation filter to changes in the speckle pattern, depends on the input radiation polarisation. In addition, diffraction from the reflection hologram in the samples of this cut is anisotropic in the general case, which leads to changes not only in the power but also in the polarisation state of the reflected light wave (Fig. 3b).

Figure 5 presents the experimental dependences of the effective gain $\Gamma_{\text {eff }}$ and the additional optical rotation $\Delta \xi_{\mathrm{R}}$ of the reflected wave on the angle $\xi_{0}$ between the polarisation plane of the input wave and the [001] axis of a $\mathrm{Ca}$, $\mathrm{Ga}: \mathrm{Bi}_{12} \mathrm{TiO}_{20}$ crystal [the (100) cut, thickness $d=8 \mathrm{~mm}$ ] used in experiments on the development of an adaptive holographic correlator.

The maximum values of $\Gamma_{\text {eff }}$ and $\Delta \xi_{\mathrm{R}}$ in this crystal were

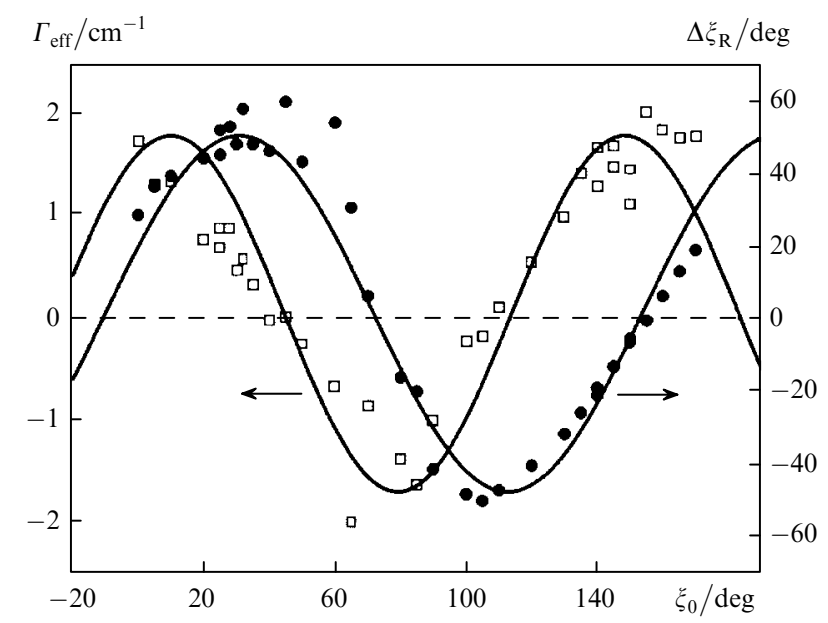

Figure 5. Experimental dependences of the effective gain $\Gamma_{\text {eff }}(\square)$ and additional optical rotation $\Delta \xi_{\mathrm{R}}(\bullet)$ of the reflected wave on the angle $\xi_{0}$ between the polarisation plane of the input wave and the [001] axis of the $\mathrm{Ca}, \mathrm{Ga}: \mathrm{Bi}_{12} \mathrm{TiO}_{20}$ crystal [the (100) cut, the thickness $d=8 \mathrm{~mm}$ ]; solid curves are the theory.
$2.1 \mathrm{~cm}^{-1}$ and $60^{\circ}$, respectively. One can see that the experimental data are in satisfactory agreement with numerical calculations based on the theoretical model considered above. As for the $\mathrm{Fe}, \mathrm{Cu}: \mathrm{Bi}_{12} \mathrm{TiO}_{20}$ crystal (Fig. 3), the maximum amplification/attenuation of the signal beam (for $\xi_{0}=150^{\circ}$ or $75^{\circ}$ ) is observed at the minimal optical rotation of this beam. At the same time, the maximum induced optical rotation of the signal beam is accompanied by virtually zero amplification (for $\xi_{0}=40^{\circ}$ and $110^{\circ}$ ). This circumstance allows one to use separately both the power modulation of the nonstationary signal light beam on the reflection grating and the modulation of its polarisation state, which can be easily recorded by using an analyser placed in front of the photodetector (see Fig. 4a).

Figure 6 presents the time dependences of the total power $P_{\mathrm{R}}$ of the beam reflected from the grating and containing the speckle structure, and the amplitude of its variation at the second harmonic frequency $\left(\Delta P_{\mathrm{R}}\right)$. Here, the increase in the amplitude of the alternate signal corresponds to the growth dynamics of the light beam power reflected from the grating, i.e. demonstrates the character of the grating formation. This confirms the fact that the power modulation of the reflected radiation is the result of interaction of the nonstationary optical field on the dynamic hologram produced by this field in the photorefractive crystal.

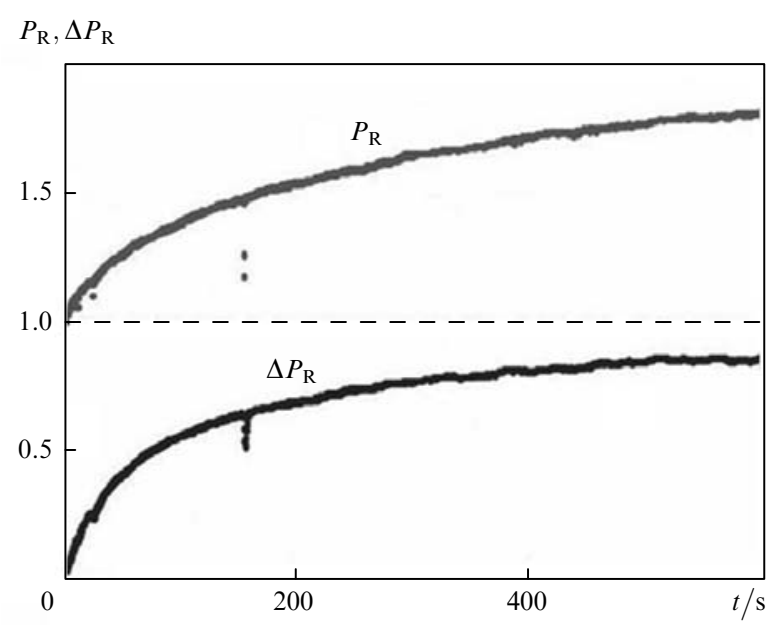

Figure 6. Time dependences of the power of the light beam $\left(P_{\mathrm{R}}\right)$ reflected from the grating and its variation amplitude at the second-harmonic frequency $\left(\Delta P_{\mathrm{R}}\right)$.

The time dependences of the amplitude of the power modulation of the reflected light wave at the first and second harmonic-frequencies of the modulated signal during the formation of the reflection grating in the crystal are presented in Fig. 7. One can see that the first-harmonic amplitude [curve (1)] achieves much smaller values compared to the second-harmonic amplitude [curve (2)] and is not stable. The presence of the first harmonic in the alternating signal is explained by the speckle modulation on the aperture of optical elements and by the random fluctuations of the speckle pattern. The stable alternating signal is observed at the second harmonic, in agreement with the conclusions made above.

To process the nonstationary field containing the speckle pattern by modulating the polarisation state of the reflected 
beam, the angle $\xi_{0}$ between the polarisation plane of the input wave and the [001] axis of the crystal was set equal to $40^{\circ}$ in accordance with the results presented in Fig. 5. In addition, variations of the signal-beam polarisation were transformed to the intensity variations by using an analyser placed in front of a photodetector. The dynamics of the power amplitude variations at the second-harmonic frequency is shown by curve (3) in Fig. 7. One can see that a stable signal exceeding the fluctuations at the first-harmonic frequency is also observed.

Thus, by using the reflection Denisyuk scheme, we performed the adaptive correlation filtration in cubic photo-

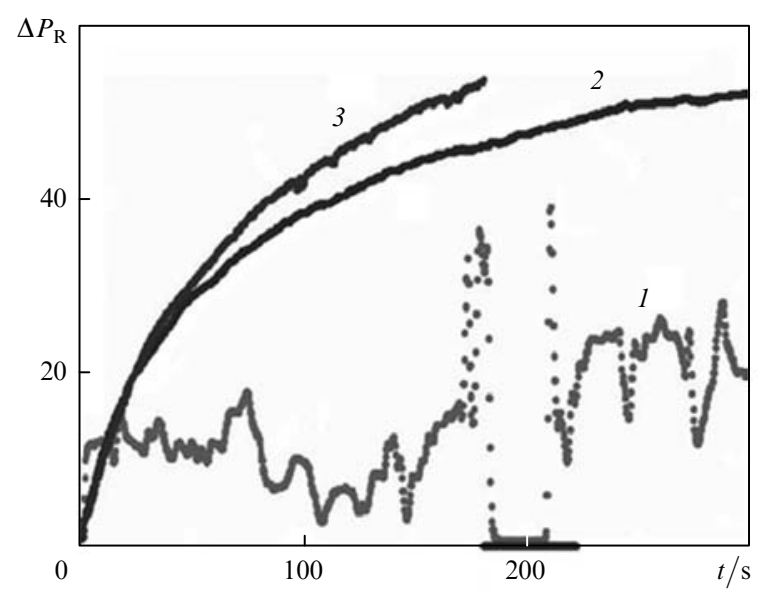

Figure 7. Time dependences of the radiation power amplitude of a light beam reflected from the grating at the first- (1) and second-harmonic $(2,3)$ frequency. refractive crystals due to the high efficiency of the hologram, which does not require the use of the external electric field. Along with the absence of the external reference wave in the scheme, this significantly simplifies the measuring system, improves its reliability and reduces the power consumption. At the same time, the transformation (doubling) of the output signal frequency negatively affects the metrological performance of the measuring system, thus limiting the scope of its possible applications. The linear regime of the phase demodulation can be achieved by using the vector wave mixing in the Denisyuk hologram.

\section{Adaptive interferometer based on the vector wave mixing in the Denisyuk hologram}

For a dynamic hologram formed by two light waves, one of which is produced due to Fresnel reflection from the crystal output face (Fig. 4a), the phase modulation in the reflected wave repeats all the phase variations of the initial wave, which makes it impossible to use this scheme as a holographic demodulator. To perform the direct phase demodulation of the light wave, it is necessary to use the reference wave, which is independent of the signal wave (Fig. 8a).

It is known that the self-diffraction of two coherent waves on the dynamic hologram formed by these waves leads to the power transfer from one wave to the other, while the phase modulation introduced into one of the waves causes the modulation of output wave power. In this case, the phase demodulation regime depends on the phase shift acquired by the wave, which is the result of diffraction from the dynamic hologram. If the phase shift for the

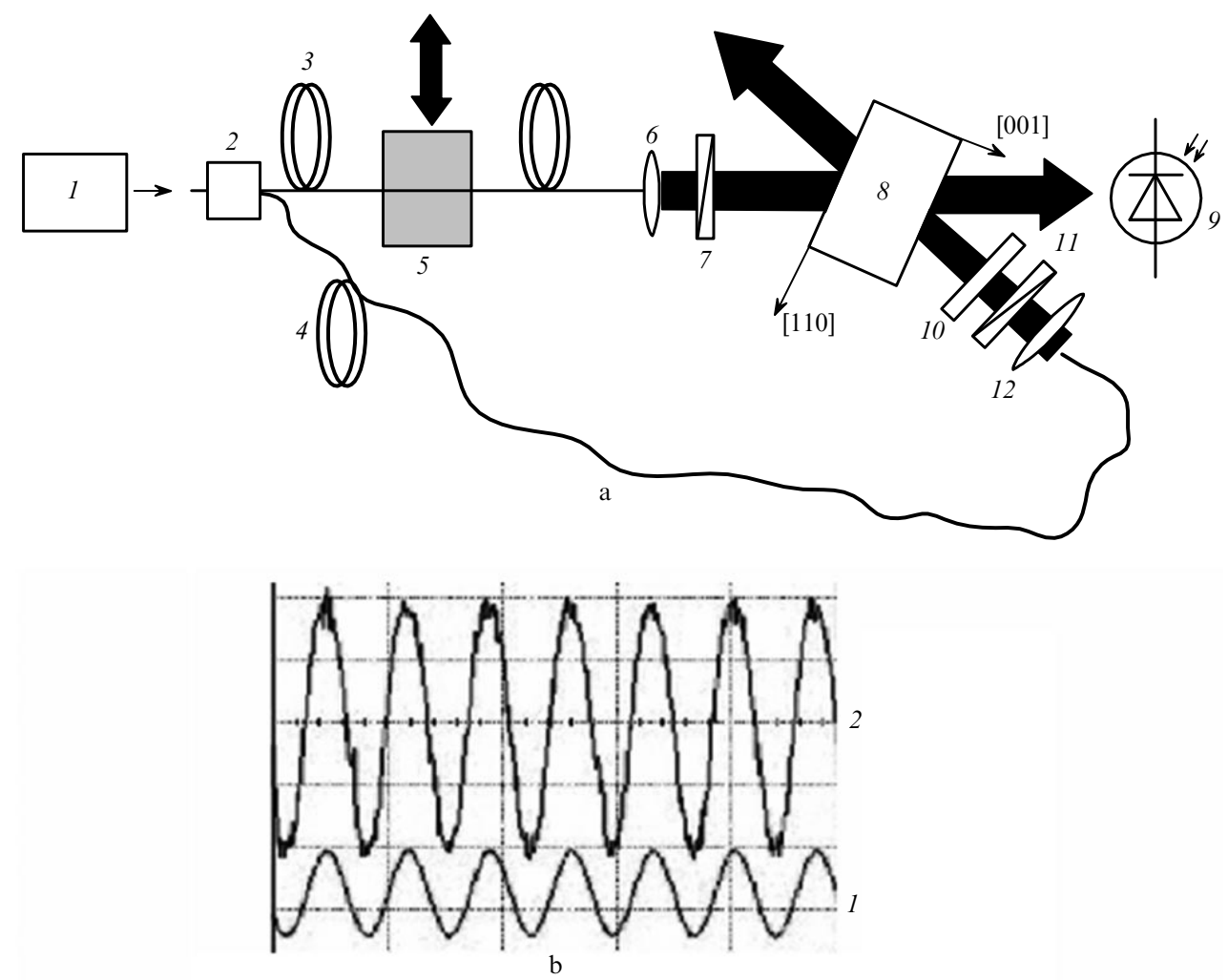

Figure 8. Scheme of the adaptive fibreoptic interferometer based on the Denisyuk hologram (a) and oscillograms of the modulation signal ( 1 ) and photodetector signal (2) (b): (1) laser; (2) optical coupler; (3,4) signal and reference optical fibres, respectively; (5) modulation action on the optical fibre; $(6,12)$ lenses; ( 7$)$ polariser; $(8)$ photorefractive crystal; (9) photodetector; (10) analyser; (11) quarter-wave plate. 
diffracted light field is equal to 0 or $\pi$, the quadratic regime is realised, the demodulation signal appearing at the secondharmonic frequency. The linear phase demodulation, when the power modulation appears at the same frequency as the phase modulation, is realised by phase shifting the diffracted field by $\pi / 2$.

The phase shift of the diffracted field, which is the sum of the phase difference between the photorefractive grating and interference pattern and the additional phase shift $-\pi / 2$ upon diffraction from the phase grating, is determined by the writing mechanism of the hologram in the photorefractive crystal. In the case of the diffusion writing mechanism (in the absence of an external electric field or in an alternating field with the switching period shorter than the hologram writing time), the total phase shift is 0 or $\pi$, which corresponds to the quadratic phase demodulation regime [1]. In the case of the drift writing, when a constant electric field is applied to the crystal, the linear regime of the phase demodulation is achieved (by providing the phase shift by $\pi / 2$ or $-\pi / 2$ ) [1]. However, a strong constant electric field applied to the crystal leads to its overheating (up to its damage). As a result, it is necessary to perform measurements within short time intervals alternated by the 'silence' periods [7]. In addition, this measuring system cannot be used for prolonged monitoring in the autonomous regime because it has the enhanced power consumption.

If the diffraction of a light wave from a dynamic hologram is anisotropic and the polarisation plane of the diffracted field is rotated by $90^{\circ}$, it is possible to achieve the linear regime of the phase modulation transformation of the signal wave to its power variation for the diffuse writing mechanism as well. The necessary condition of the linear phase demodulation is the mixing of two waves with different polarisations: one wave should be linearly polarised and the other - elliptically polarised. The demodulation linearity is achieved due to the fact that the internal phase difference in $\pi / 2$ between the orthogonal components of the elliptically polarised wave provides an additional phase shift between the transmitted signal wave and the reference wave field. The linear phase demodulation regime realised due to the vector mixing of waves with different polarisations in the diffusion hologram in the transmission geometry was first experimentally demonstrated in paper [51]. Later, this approach was realised in the reflection geometry of the dynamic hologram formation shown in Fig. 8a [52]. Here, the signal wave is linearly polarised and the reference wave is elliptically polarised. Based on the rigorous theory of the vector wave mixing [53], it was shown that in the geometry under study the linear phase demodulation regime is achieved, which is illustrated by the oscillograms of the phase modulation signals and by changes in the optical power directly measured by the photodetector (Fig. 8b).

It is known that a classical homodyne interferometer operating in the quadrature regime has the maximum possible sensitivity to phase variations [7]. For an adaptive interferometer based on the diffusion writing of a dynamic hologram, the ratio $\delta_{\text {rel }}$ of the minimum fixed phase modulation to the minimum modulation detected by the classical interferometer is determined by the expression [54]:

$$
\delta_{\text {rel }}=\sqrt{2} \exp \left(\frac{\alpha d}{2}\right) \frac{P_{\mathrm{S}}}{\Delta P_{\mathrm{S}}} \Phi_{0}
$$

where $P_{\mathrm{S}}$ and $\Delta P_{\mathrm{S}}$ are the power and amplitude of the signal wave power modulation, respectively, and $\Phi_{0}$ is the phase modulation amplitude of the signal light wave.

The sensitivities of the reflection and transmission schemes can be compared by studying the dependence of the relative detection limit on the spatial period $\Lambda$ of the holographic grating formed in the photorefractive crystal. Figure 9 shows the dependences $\delta_{\text {rel }}(\Lambda)$ obtained in [54] for two cubic $\mathrm{V}: \mathrm{CdTe}$ crystals in which photorefractive holograms were written by IR radiation at $1064 \mathrm{~nm}$. One can see that, as the holographic grating period was decreased from 5.2 to $0.8 \mu \mathrm{m}$ in the transmission geometry, the relative detection range decreased; however, its minimum value for each of the crystals was achieved only in the reflection geometry $(\Lambda=0.19 \mu \mathrm{m})$. As mentioned above, the high efficiency of the reflection hologram (and, hence, the high sensitivity of the adaptive interferometer based on it) can be achieved only if the concentration of photoactive traps is high enough. This concentration in case under study can be estimates as $N_{\mathrm{a}} \geqslant 2 \times 10^{22} \mathrm{~m}^{-3}$.

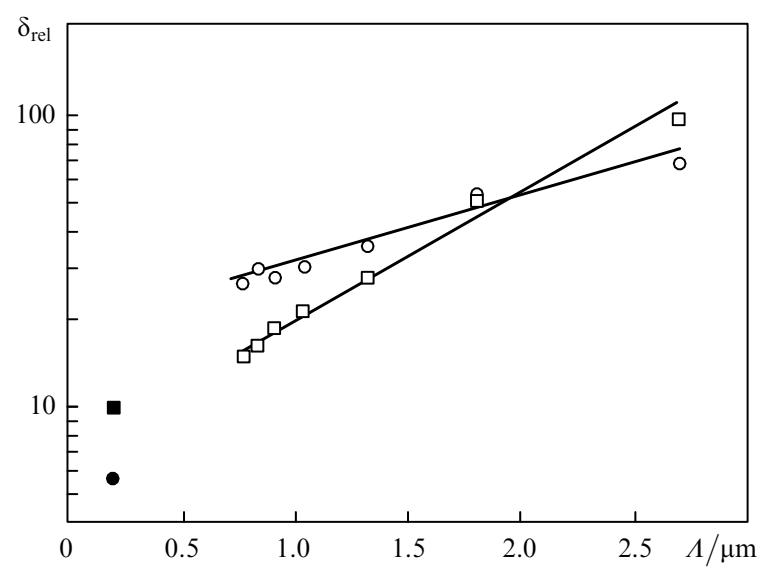

Figure 9. Dependences of the relative detection limit on the holographic grating period for the $\mathrm{V}: \mathrm{CdTe}$-crystal samples in the transmission writing geometry [sample $1(\circ)$ and sample $2(\square)$ ] and in the reflection writing geometry [sample $1(\bullet)$ and sample $2(\boldsymbol{\bullet})$ ].

One can see that the sensitivity of the adaptive interferometer based on the diffusion Denisyuk hologram is only 5.7 times smaller than that of the classical homodyne interferometer, which has no optical losses and is not adaptive.

\section{Conclusions}

We have considered papers devoted to the theoretical analysis, experimental studies and application of dynamic Denisyuk holograms written in cubic photorefractive crystals in adaptive measuring systems. The history of the development of studies on reflection holograms in cubic photorefractive crystals has been outlined. The results of measurements of optimal polarisations of interacting waves and optimal crystal cuts obtained by various researchers have been presented. It has been shown that in the general case for the crystal thickness larger than the critical one, the (001) and (111) cuts are not optimal. The equations for arbitrarily polarised coupled waves have been considered which describe the interaction of counterpropagating waves 
on reflection gratings produced due to the diffusion mechanism in cubic photorefractive crystals of the arbitrary cut, which show in the general case the natural optical activity. The results of the analysis of the two-beam interaction of linearly polarised waves on reflection photorefractive gratings have been presented. It has been pointed out that in the (100)-cut bismuth titanate crystals of thickness smaller than $10 \mathrm{~mm}$, the effective gain in the interaction of two counterpropagating waves can achieve $7 \mathrm{~cm}^{-1}$ without applying the external electric field.

The application of the Denisyuk reflection scheme for realising adaptive elements in measuring systems based on optical and fibreoptic interferometers has been demonstrated. Such measuring systems can be successfully used under real conditions due to their ability to adapt to the uncontrollable variations in the environment. We have considered the schemes of interaction of two counterpropagating waves on reflection holograms in the $\mathrm{Ca}, \mathrm{Ga}: \mathrm{Bi}_{12} \mathrm{TiO}_{20}$ crystal, which realise the adaptive correlation filter, and the adaptive interferometer based on the vector wave mixing. The experimental dependence of the relative detection limit of the holographic interferometer on the spatial period of the photorefractive grating produced in $\mathrm{V}: \mathrm{CdTe}$ crystals has been presented, which demonstrates the advantages of the Denisyuk scheme over the conventional transmission scheme for writing photorefractive holograms.

Acknowledgements. This work was supported by the Russian Foundation for Basic Research (Grant Nos 0602-81040_Bel_a and 07-02-91015), 'The Development of the Scientific Potential of the Higher School 2006-2008)' program and the Ministry of Education of the Republic of Belarus (SCPSR 'Photonics').

\section{References}

1. Petrov M.P., Stepanov S.I., Khomenko A.V. Fotorefractivnye kristally $v$ kogerentnoi optike (Photorefractive Crystals in Coherent Optics) (St. Petersburg: Nauka, 1992).

2. Odulov S.G., Soskin M.S., Khizhnyak A.I. Lazery na dinamicheskikh reshetkakh: opticheskie generatory na chetyrekhvolnovom smeshenii (Dynamic-grating Lasers: Fourwave-mixing Optical Generators) (Moscow: Nauka, 1990).

3. Yeh P. Introduction to Photorefractive Nonlinear Optics (New York: Wiley, 1993).

4. Stepanov S.I. Applications of Photorefractive Crystals (Repts. Progr. Phys., 1994) Vol. 57, pp 39-116.

5. Solymar L., Webb D.J., Grunnet-Jepsen A. The Physics and Application of Photorefractive Materials (Oxford: Clarendon Press, 1996).

6. Kamshilin A.A., Jaaskelainen T., Kulchin Yu.N. Appl. Phys. Lett., 73, 705 (1998).

7. Delaye P., Blouin A., Drolet D., De Montmorillon L.-A., Roosen G., Monchalin J.-P. J. Opt. Soc. Am. B, 14, 1723 (1997).

8. Kamshilin A.A., Prokofiev V.V. Opt. Lett., 27, 1711 (2002)

9. Petrov M.P., Bryksin V.V., in Photorefractive Materials and their Applications 2. Ed. by P. Gunter, J.P. Huignard (Berlin: Springer-Verlag, 2007) pp 285-325.

10. Denisyuk Yu.N. Dokl. Akad. Nauk SSSR, 144, 1275 (1962).

11. Denisyuk Yu.N. Opt. Spektrosk., 15, 522 (1963).

12. Ja Y.H. Opt. Quantum Electron., 14, 547 (1982).

13. Yeh P. J. Opt. Soc. Am., 73, 1268 (1983).

14. Yeh P. Appl. Opt., 23, 2974 (1984)

15. Ja Y.H. Opt. Quantum. Electron., 16, 399 (1984).

16. Erbschloe D., Solymar L., Takacs J., Wilson T. IEEE J. Quantum Electron., 24, 820 (1988).
17. Kukhtarev N.V., Dovgalenko G.E., Starkov V.N., Appl. Phys. A, 33, 227 (1984).

18. Khramovich E.M., Shepelevich V.V. Izv. Akad. Nauk BSSR. Ser. Fiz.-Mat. Nauk, (2), 106 (1987).

19. Kukhtarev N., Chen Bo Su, Venkateswarlu P., Salamo G., Klein M. Opt. Commun., 104, 23 (1993).

20. Dovgalenko G., Salamo G., Duree G., et al., in Digest of Topical Meeting on Photorefractive Materials, Effects and Devices (PRM'95) (Washington: OSA, 1995) p. 295.

21. Miteva M., Nikolova L. J. Modern. Opt., 43, 1801 (1996).

22. Mallick S., Miteva M., Nikolova L. J. Opt. Soc. Am. B, 14, 1179 (1997).

23. Mart'yanov A.G., Shandarov S.M., Litvinov R.V. Fiz. Tverd. Tela, 44, 1006 (2002).

24. Stepanov S.I., Shandarov S.M., Khat'kov N.D. Fiz. Tverd. Tela, 29, 3054 (1987).

25. Shepelevich V.V., Shandarov S.M., Mandel A.E. Ferroelectrics, 110, 235 (1990).

26. Volkov V.I., Kargin Yu.F., Kukhtarev N.V., Privalko A.V., Semenets T.I., Shandarov S.M., Shepelevich V.V. Kvantovaya Elektron., 18, 1237 (1991) [Sov. J. Quantum Electron., 21, 1122 (1991)].

27. Shandarov S.M., Shepelevich V.V., Khat'kov N.D Opt. Spektrosk., 70, 1068 (1991) [Opt. Spectrosc. (USSR), 70, 627 (1991)].

28. Ageev E.Yu., Shandarov S.M., Veretennikov S.Yu., Mart'yanov A.G., Kartashov V.A., Kamshilin A.A., Prokof'ev V.V., Shepelevich V.V. Kvantovaya Elektron., 31, 343 (2001) [Quantum Electron., 31, 343 (2001)].

29. Mart'yanov A.G., Ageev E.Yu., Shandarov S.M., Mandel' A.E., Bochanova N.V., Ivanova N.V., Kargin Yu.F., Volkov V.V., Egorysheva A.V., Shepelevich V.V. Kvantovaya Elektron., 33, 226 (2003) [Quantum Electron., 33, 226 (2003)].

30. Martyanov A.G., Antonova N.Yu., Shandarov S.M., Kargin Yu.F., Volkov V.V., Egorysheva A.V., Prokofiev V.V., in ICO Topical Meeting on Polarization Optics (Polvijarvi, Finland, 2003) p. 220.

31. Plesovskikh A.M., Shandarov S.M., Mart'yanov A.G., Mandel' A.E., Burimov N.I., Shaganova E.A., Kargin Yu.F., Volkov V.V., Egorysheva A.V. Kvantovaya Elektron., 35, 163 (2005) [ Quantum Electron., 35, 163 (2005)].

32. Shepelevich V.V., Navnyko V.N., Nichiporko S.F. Shandarov S.M., Mandel' A.E. Pis'ma Zh. Tekhn. Fiz., 29 (18), 22 (2003) [Techn. Phys. Lett., 29 (9), 757 (2003)].

33. Lichtenberg S., Petrov V.M., Petter J., Tschudi T., Chamrai A.V., Petrov M.P. Ukr. J. Phys., 49, 467 (2004).

34. Shepelevich V.V., Navnyko V.N. Pis'ma Zh. Tekhn. Fiz., 33 (17), 16 (2007) [Techn. Phys. Lett., 33 (9), 726 (2007)].

35. Shepelevich V.V., Navnyko V.N. Vestnik MozGPU, (1), 21 (2006).

36. Shepelevich V.V., Navnyko V.N. Izv. GGU im. F. Skariny, 6, 143 (2006).

37. Weber M., Shamonina E., Ringhofer K.H., von Bally G. Opt. Mater., 18, 119 (2001).

38. Von Bally G., Thien R., Kemper B. Ukr. J. Phys., 49, 457 (2004).

39. Romashko R.V., Shandarov S.M., Kul'chin Yu.N., Burimov N.I. Limarev D.A., Kargin Yu.F., Volkov V.V. Izv. Ros. Akad. Nauk. Ser. Fiz., 69, 1143 (2005).

40. Hahn J., Petrov V., Petter J., Tschudi T., Petrov M. OSA Trends in Optics and Photonics (TOPS) (Washington, DC: OSA, 2003) Vol. 99, pp 669-674.

41. Petrov V., Hahn J., Petter J., Petrov M., Tschudi T. Opt. Lett., 30, 3138 (2005).

42. Shandarov S.M., Burimov N.I., Dubtsov M.A., Sibagatulina V.G., Baklanov D.S., Kulchin Yu.N., Romashko R.V., Kargin Yu.F., Egorysheva A.V., Volkov V.V Laser Phys., 17, 482 (2007).

43. Kukhtarev N.V., Markov V.B., Odulov S.G., Soskin M.S., Vinetskii V.L. Ferroelectrics, 22, 949 (1979).

44. Tayebati P., Mahgerefteh D. J. Opt. Soc. Am. B, 8, 1053 (1991).

45. Veretennikov S.Yu., Mandel' A.E., Shandarov S.M. Kazarin A.V., Tsurkan M.I., Plesovskikh A.M., Egorysheva A.V., 
Kargin Yu.F., Bikeev O.N., Shepelevich V.V. Izv. Vyssh. Uchebn. Zaved. Ser. Fiz., 46 (2), 39 (2003).

46. Kargin Yu.F., Egorysheva A.V., Volkov V.V., Burkov V.I., Shandarov S.M., Mandel A.E., Skorikov V.M. J. Crystal Growth., 275, 779 (2005).

47. Hall T.J., Fiddy M.A., Ner M.S. Opt. Lett., 5, 485 (1980)

48. Huignard J.P., Marrakchi A. Opt. Lett., 6, 622 (1981).

49. Stepanov S.I. International Trends in Optics. Ed. by

J.W. Goodmen (New York, London: Acad. Press Inc.) Ch. 9.

50. Romashko R.V., Kulchin Yu.N., Shandarov S.M., Kargin Yu.F., Volkov V.V. Opt. Rev., 12, 58 (2005).

51. Kamshilin A.A., Grachev A.I. Appl. Phys. Lett., 81, 2923 (2002).

52. Romashko R.V., Kul'chin Yu.N., Kamshilin A.A. Izv. Ros. Akad. Nauk. Ser. Fiz., 70, 1296 (2006).

53. Sturman B.I., Podivilov E.V., Ringhofer K.H., Shamonina E., Kamenov V.P., Nippolainen E., Prokofiev V.V., Kamshilin A.A. Phys. Rev. E, 60, 3332 (1999).

54. Di Girolamo S., Kamshilin A.A., Romashko R.V., Kulchin Yu.N., Launay J.-C. Opt. Express, 15, 545 (2007). 